\title{
Modeling of a low-loss spot-size converter for hollow waveguides with sub-wavelength air core
}

\author{
Yasuki Sakurai ${ }^{\mathrm{a})}$ and Fumio Koyama \\ Microsystem Research Center, Tokyo Institute of Technology \\ 4259-R2-22 Nagatsuta, Midori-ku, Yokohama 226-8503, Japan \\ a)ysakurai@ms.pi.titech.ac.jp
}

\begin{abstract}
We propose the integration of a tapered spot-size converter for coupling between a narrow air-core hollow waveguide and a single-mode fiber (SMF). The linear taper waveguide consisting of $\mathrm{SiO}_{2}$ is integrated with a hollow waveguide composed of highly reflective multilayer mirrors. Light is guided with total internal reflections at a $\mathrm{SiO}_{2}$ taper surface and the multiple reflections in the multilayer mirror. The core thickness of a taper $\mathrm{SiO}_{2}$ waveguide is linearly changed from $15 \mu \mathrm{m}$ to $0.6 \mu \mathrm{m}$. The modeling result shows a coupling loss of less than $1 \mathrm{~dB}$ between a SMF and a hollow waveguide with an air-core thickness of $1 \mu \mathrm{m}$. A taper waveguide-induced loss is less than $0.1 \mathrm{~dB}$ for a two-dimensional linear taper waveguide with a length of less than $1 \mathrm{~mm}$. Using the proposed structure, we can expect the dramatic reduction in coupling losses by $10 \mathrm{~dB}$. The device structure can be formed by fully planar fabrication processes based on lithography, etching and coating. The proposed spot-size converter enables us to achieve a large tunability of over $10 \%$ in propagation constant in hollow waveguides with a variable air-core.
\end{abstract}

Keywords: hollow waveguide, air-core, spot-size converter, taper waveguide, coupling

Classification: Photonics devices, circuits, and systems

\section{References}

[1] T. Miura, F. Koyama, and A. Matsutani, "Modeling and Fabrication of Hollow Optical Waveguide for Photonic Integrated Circuits," Jpn. J. Appl. Phys., vol. 41, no. 7B, pp. 4785-4789, July, 2002.

[2] Y. Sakurai and F. Koyama, "Proposal of Tunable Hollow Waveguide Distributed Bragg Reflectors," Jpn. J. Appl. Phys., vol. 43, no. 5A, pp. L631L633, April, 2004.

[3] Y. Sakurai and F. Koyama, "Control of Group delay and Chromatic Dispersion in Tunable Hollow Waveguide with Highly Reflective Mirrors," submitted to Jpn. J. Appl. Phys., vol. 43, no. 8B, 2004.

[4] T. Shoji, T. Tsuchizawa, T. Watanabe, K. Yamada, and H. Morita, "Low loss mode size converter from $0.3 \mu \mathrm{m}$ square Si wire waveguides to singlemode fibers," Electron. Lett., vol. 38, no. 25, pp. 1669-1670, Dec., 2002. 
[5] P. Sanchis, J. Marti, A. Garcia, A. Martinez, and J. Blasco, "High efficiency coupling technique for planar photonic crystal waveguides," Electron. Lett., vol. 38, no. 17, pp. 961-962, Aug., 2002.

\section{Introduction}

Various tunable optical devices are needed for the realization of future photonic networks including ultra-dense wavelength division multiplexing, all optical signal processing, ultrahigh bit-rate signal processing and so on. We proposed hollow waveguide tunable devices with a variable air core, which may have unique features such as temperature insensitive operation and wide tunability of propagation constant more than $10 \%[1,2]$. We predicted a large chromatic dispersion of more than $100 \mathrm{ps} / \mathrm{nm} / \mathrm{cm}$ and slow light with a reduced velocity of 10 times lower than that in vacuum using a hollow waveguide with a sub-micron air-core [3]. A hollow waveguide with a narrow air-core enables a continuous wide tuning of propagation constant, chromatic dispersion and group velocity. A difficulty will be a large coupling loss between a narrow air-core and a single-mode fiber (SMF). Thus, a spot-size converter for a hollow waveguide with a narrow air-core will be useful for realizing the unique features of tunable hollow waveguides.

Recently, low-loss coupling with a coupling loss of less than $1 \mathrm{~dB}$ has been demonstrated in Si wire or photonic crystal waveguides with a submicron core using a spot-size converter $[4,5]$. In this paper, we propose the integration of a tapered spot-size converter for low-loss coupling between a narrow air-core hollow waveguide and SMF. Using the proposed spotsize converter with a taper length of less than $1 \mathrm{~mm}$, we show the dramatic reduction in coupling losses by $10 \mathrm{~dB}$ in comparison with that in butt-coupling between $\mathrm{SMF}$ and a $1 \mu \mathrm{m}$ air-core hollow waveguide.

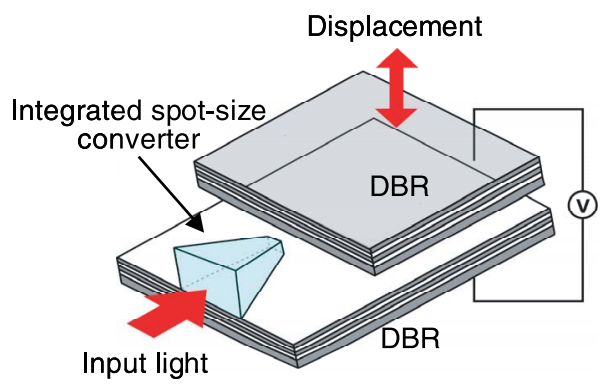

Bird's-eye view

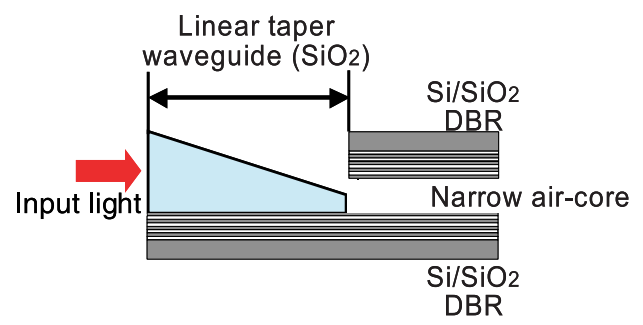

Side view

Fig. 1. Schematic structure of spot-size converter integrated hollow waveguide with variable narrow aircore. 


\section{Structure of spot-size converter for hollow waveguides with a narrow air-core}

Figure 1 shows the schematic structure of our proposed spot-size converter for hollow waveguides with a narrow air-core. The linear taper waveguide consisting of $\mathrm{SiO}_{2}$ is loaded on the input side of a hollow waveguide composed of highly reflective multilayer mirrors (DBR).

Light is guided with total internal reflections at a taper surface and the multiple reflections in the multilayer mirror. To reduce Fresnel reflection losses due to the high index contrast at each boundary, we chosen $\mathrm{SiO}_{2}$ as taper waveguide material. The device structure can be formed by fully planar fabrication processes based on deposition, lithography and etching. We chosen $\mathrm{Si} / \mathrm{SiO}_{2}$ multilayer stack mirrors as hollow waveguide clad for modeling of a hollow waveguide. In this multilayer structure, we can expect the strong confinement in a narrow air-core with a help of highly reflective multilayers having a small numbers of pairs due to their high index contrast.

\section{Coupling loss characteristics}

In the numerical modeling, we assume 10 pair $\mathrm{Si} / \mathrm{SiO}_{2}$ multilayer stack with refractive indices of $\mathrm{Si}$ and $\mathrm{SiO}_{2}$ to be 3.5 and 1.44, respectively. Each layer is designed to be a quarter wavelength for oblique incident angles. A taper waveguide consists of $\mathrm{SiO}_{2}$ with a refractive index of 1.44. The wavelength in the numerical simulation is $1550 \mathrm{~nm}$ for TE mode.

First, we calculated the coupling losses between SMF and the input taper waveguide, the output taper waveguide and the hollow waveguide with an aircore thickness of $1 \mu \mathrm{m}$ in order to optimize the core thickness of the taper waveguide. We carried out 2-dimensional full-vectorial simulation by using a simulator (FIMMWAVE, provided by Photon Design Company), which is based on a film-mode-matching method. The core thicknesses of each end of the taper waveguide, which minimize the coupling loss at each boundary, are $15 \mu \mathrm{m}$ and $0.6 \mu \mathrm{m}$, respectively. The coupling losses for the SMF and the hollow waveguide are $0.064 \mathrm{~dB}$ and $0.78 \mathrm{~dB}$, respectively. Calculated intensity distributions in a vertical direction of a substrate at each waveguide section are almost matched with each other as shown in Fig. 2.

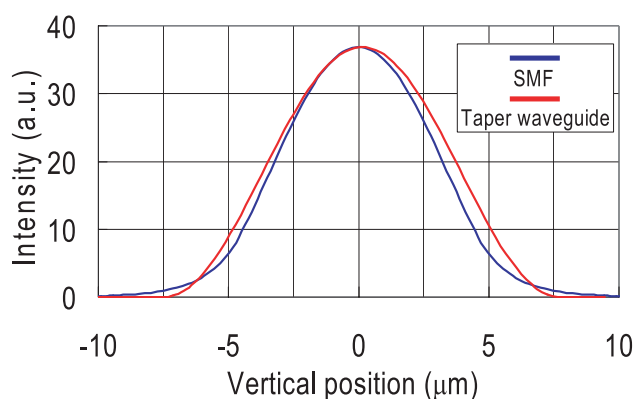

(a) Input taper waveguide boundary

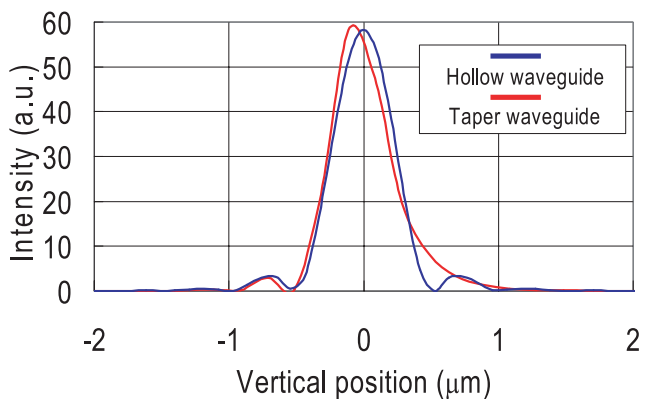

(b) Output taper waveguide boundary

Fig. 2. Mode field distribution at each waveguide section. 
Also, we calculated the coupling loss between a SMF and a hollow waveguide with an air-core thickness of $1 \mu \mathrm{m}$ as a function of the taper length on a taper spot-size converter as shown in Fig. 3(a). In this calculation, we assume a two-dimensional taper waveguide with a core thickness linearly changed from $15 \mu \mathrm{m}$ to $0.6 \mu \mathrm{m}$ in a vertical direction. In this modeling, we carried out two-dimensional waveguide simulation corresponding to the coupling between a tapered slab waveguide and a slab hollow waveguide.

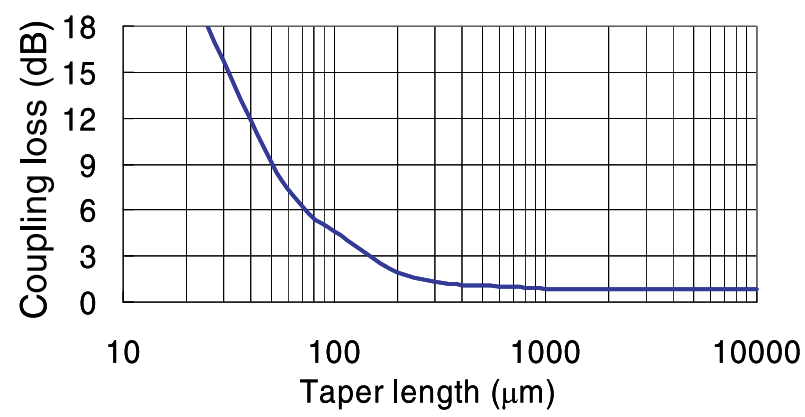

(a)

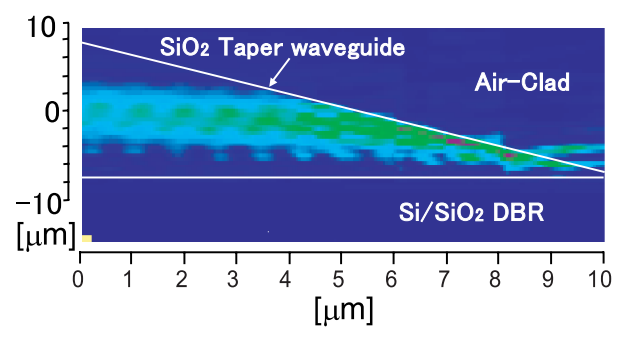

Taper length $10 \mu \mathrm{m}$

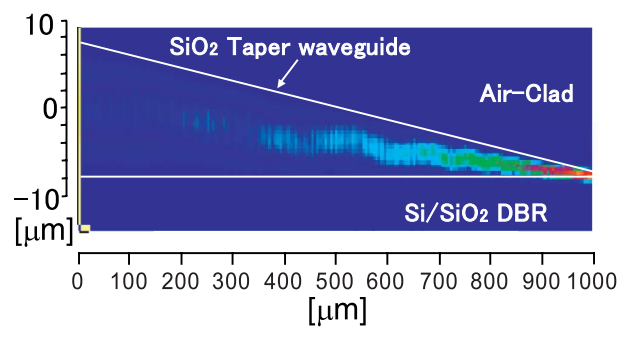

Taper length $1 \mathrm{~mm}$

(b)

Fig. 3. (a) The coupling loss between a SMF and a hollow waveguide with an air-core thickness of $1 \mathrm{~mm}$ versus taper waveguide length and (b) intensity distribution in a linear taper $\mathrm{SiO}_{2}$ waveguide.

The coupling loss is less than $1 \mathrm{~dB}$ with a taper length of more than $700 \mu \mathrm{m}$. A taper waveguide-induced excess loss with a taper length of $1 \mathrm{~mm}$ is less than $0.1 \mathrm{~dB}$. Using this structure, we can expect the reduction in coupling loss by $10 \mathrm{~dB}$ in comparison with that in the case of butt-coupling between a SMF and a hollow waveguide with an air-core thickness of $1 \mu \mathrm{m}$.

Figure 3 (b) shows the intensity distribution in a linear taper $\mathrm{SiO}_{2}$ waveguide. The taper waveguide with a short taper length such as $10 \mu \mathrm{m}$ causes noticeable reflections at a taper surface and radiation through an air-clad because of the steep taper angle of a tapered $\mathrm{SiO}_{2}$ waveguide. On the other hand, a $1 \mathrm{~mm}$ long taper waveguide enables us to convert smoothly the mode field of SMF into the fundamental guided mode field of a hollow waveguide with an air-core thickness of $1 \mu \mathrm{m}$. 


\section{Conclusion}

We proposed the integration of a tapered spot-size converter for low-loss coupling between a SMF and a hollow waveguide with a narrow air-core. The modeling result shows a coupling loss of less than $1 \mathrm{~dB}$ between a SMF and a hollow waveguide with an air-core thickness of $1 \mu \mathrm{m}$ by using a linear taper slab $\mathrm{SiO}_{2}$ waveguide with a length of less than $1 \mathrm{~mm}$. The device structure would be formed by fully planar fabrication processes based on deposition, lithography and etching. The proposed concept may open up a new class of widely tunable hollow waveguide devices including tunable filters, lasers and dispersion compensators. Low-loss coupling into a narrow air-core may enable us to achieve unique features of hollow waveguides such as giant tunability, large chromatic dispersion and slow light.

\section{Acknowledgments}

The authors acknowledge Professor Emeritus Kenichi Iga of Tokyo Institute of Technology for his encouragement. This work was supported by the Grant-in-Aid for Creative Scientific Research from the Ministry of Education, Science, Sport and Culture (\#14GS0212"). 